\title{
LÍDERES POLITICAS ARGENTINAS EN TWITTER. UN ESTUDIO EN EL MARCO DE LAS ELECCIONES 2017
}

\author{
Dr. Esteban Zunino \\ CONICET, Mendoza, Argentina \\ estebanzunino@hotmail.com \\ Dra. Lorena Recalde Cerda \\ Escuela Politécnica Nacional de Ciencias de la computación, Quito, Ecuador \\ Lorena.recalde@epn.edu.ec \\ Dra. Gabriela Baquerizo \\ Universidad Casa Grande, Guayaquil, Ecuador \\ gbaquerizo@casagrande.edu.ec
}

Recibido el 8 de abril de 2019

Aceptado el 14 de junio de 2019

\section{Resumen}

El uso de las redes sociales en las campañas electorales marca el pulso de las estrategias actuales de la comunicación política. La conversación virtual de los candidatos promueve definiciones de los problemas públicos que pretenden operar sobre la actitud hacia el voto de los ciudadanos. El presente estudio analiza el uso de Twitter por parte de las principales líderes políticas argentinas durante la campaña de las elecciones legislativas de medio término de 2017. Específicamente, se pretende investigar cuáles fueron los temas promovidos por las lideresas, qué tipo de interacción mantuvieron con los medios "tradicionales" y qué diálogos establecieron con los ciudadanos. Los resultados demuestran que las conversaciones en Twitter se centraron en las consignas de campaña, incluyeron información ligada a la dimensión local y no abundaron en temáticas relacionadas al género. En un contexto en que los temas ligados a la mujer cobraron relevancia en las agendas política, mediática y pública, este trabajo pretende ir más allá del uso político de Twitter para identificar rasgos particulares de la construcción del liderazgo femenino en las redes sociales en contextos electorales.

Palabras clave: mujeres, política, Twitter, Argentina, elecciones, género. 


\title{
ARGENTINE POLITICAL LEADERS ON TWITTER. A STUDY ABOUT THE MIDTERM ELECTIONS IN 2017
}

\begin{abstract}
The use of Online Social Networks (OSNs) in electoral campaigns is no longer an option for candidates and their campaign teams. Their performance in the virtual public sphere will mark substantial differences at the time of vote. Indeed, although the presence of politicians in social media does not guarantee their victory in elections, it is unthinkable to build their public profile outside of them. The present study intends to analyze the use of Twitter by the main female Argentinian political leaders during the campaign of the mid-term legislative elections in Argentina in 2017. Specifically, it is intended to investigate which were the topics promoted by these leaders in the public virtual sphere and what kind of dialogues they established with citizens and between them. Likewise, based on their interaction in social networks, an attempt will be made to identify which leadership characteristics these candidates possess. Finally, the aim is to analyze the influence of the gender variable on its narratives. Results show that the conversations focused on campaign slogans, they included information linked to the local dimension and did not abound on gender-related issues. In a context where issues related to women became relevant in the political, media and public agendas, this work aims to go beyond the political use of Twitter to identify particular features of the construction of female leadership in social networks.
\end{abstract}

Keywords: women, politics, Twitter, Argentina, elections, gender. 
Introducción

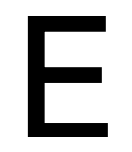

I uso de redes sociales en campañas electorales ya no es una opción para los candidatos y sus equipos. Su desempeño en la esfera pública virtual marcará diferencias sustanciales a la hora del voto, ya que, si bien la presencia de los políticos en las redes sociales no gana una elección per se, resulta impensable resultar victoriosos fuera de ellas (D’Adamo, García y Kievsky, 2015).

El presente estudio analiza el uso de la red social Twitter por parte de las principales líderes políticas ${ }^{1}$ argentinas durante la campaña de las elecciones legislativas de medio término de 2017. Concretamente, se investiga cuáles fueron los temas que promovieron las dirigentes en la esfera pública virtual (Habermas, 2006), qué tipo de interacción mantuvieron con los medios "tradicionales" y qué diálogos establecieron con los ciudadanos y entre ellas. Asimismo, a partir de su interacción en redes sociales, se procurará identificar qué características de liderazgo poseen estas candidatas. Finalmente, se analiza la influencia de la variable género sobre sus narrativas.

En la Argentina el uso de internet presenta uno de los niveles más altos de la región. Según la Encuesta de Consumos Culturales 2017, el 97\% de las personas menores de 29 años se conectan a la web cotidianamente, aunque los porcentajes también son importantes entre los ciudadanos de más edad, exhibiendo promedios de conexión del 90,4\% entre 30 y 49 años, del 70,6\% entre 50 y 64 y del $34,1 \%$ en los mayores de 65 años. Entre los principales consumos en internet, las redes sociales se destacan. Si bien Facebook domina la escena con un 64,4\% de penetración, la cantidad de cuentas de Twitter es importante, alcanzando a un 13,2\% de la población (SINCA, 2017).

La riqueza de la red social Twitter para el análisis radica en dos factores determinantes. En primer lugar, varios trabajos han demostrado que su propia fisonomía, tanto estructural como de uso, hace converger en la dinámica tuitera a los principales actores sociales, políticos y económicos (líderes políticos, medios de comunicación, periodistas, líderes de opinión, corporaciones y ciudadanos, entre otros) (Amado y Tarullo, 2015; Calvo, 2015; Kwak et al., 2010; Vargo et al., 2014). En segundo lugar, Twitter posee una Interface para Programas de Aplicación (API) que permite ser asociada a diferentes programas de software con el fin de recuperar y sistematizar datos de contenido y navegación libremente, lo

\footnotetext{
1 El líder político puede caracterizarse como un sujeto que ejerce poder, influye y moviliza a sus "seguidores" -en redes sociales- con la finalidad de lograr metas comunes (Heifetz, 1997; Lupano y Castro, 2008; Zalles, 2011).
} 
que, a diferencia de Facebook, por ejemplo, posibilita acceder públicamente a una serie de datos con el fin de reconstruir trayectorias y mapas de interacción.

El contexto en el que se llevó a cabo la investigación fue el de las elecciones legislativas 2017 en la Argentina. En esa ocasión, la alianza de gobierno encabezada por Mauricio Macri (Cambiemos) plebiscitaba su gestión enfrentando a diferentes espacios políticos, aunque polarizaba centralmente con la figura de la expresidenta Cristina Fernández de Kirchner (2007-2015). Con ese condimento, la contienda marcaría el pulso tanto de la vigencia del programa de gobierno de Cambiemos, como de la capacidad de la oposición de enfrentarse al Poder Ejecutivo de manera exitosa.

La particularidad del caso es que diferentes lideresas jugaron papeles fundamentales, ya sea a través de candidaturas, o bien a partir de la ostentación de cargos ejecutivos desde los cuales se convirtieron en actrices centrales de las campañas de cada espacio. Cristina Fernández de Kirchner fue candidata en la Provincia de Buenos Aires, Elisa Carrió (Cambiemos) en la Ciudad de Buenos Aires $^{2}$ y la principal coalición de izquierda, el Frente de Izquierda y los Trabajadores (FIT), también llevó en sus listas candidatas mujeres.

En este trabajo se analizó el uso de Twitter por parte de lideresas en campañas de diferentes escalas y dimensiones territoriales. Para ello, se compararon a las dirigentes nacionales Elisa Carrió (Cambiemos), Cristina Fernández (Unidad Ciudadana), María Eugenia Vidal (Cambiemos) y Miriam Bregman (FIT), con las lideresas políticas de la provincia de Mendoza ${ }^{3}$ Claudia Najul (Cambiemos)، Cecilia Juri (Unidad Ciudadana), Noelia Barbeito (FIT) y Patricia Fadel (Partido Justicialista - Unidad Ciudadana). De este modo, una última preocupación de este trabajo es determinar si la variable geográfica afectó el desempeño de las políticas en la red social.

\section{Las elecciones legislativas 2017}

La contienda electoral legislativa de 2017 tenía el condimento de plebiscitar el gobierno de Mauricio Macri (2015-2019). La primera instancia se llevó cabo el 23 de agosto, ocasión en que se desarrollaron las Primarias Simultáneas Abiertas y Obligatorias (PASO), elección de la cual surgieron las propuestas definitivas de cada espacio político.

En la Ciudad de Buenos Aires la dirigente Elisa Carrió (Vamos Juntos Cambiemos) enfrentaba al dirigente kirchnerista Daniel Filmus (Unidad Porteña). En el mismo ámbito, la candidata nacional más importante del FIT, Miriam Bregman, apuntaba a una banca en la Legislatura de la Ciudad de Buenos Aires.

2 La Ciudad Autónoma de Buenos Aires es la Capital de la República Argentina.

3 Mendoza es representa al cuarto conglomerado urbano a nivel nacional. 
En la provincia de Buenos Aires, en tanto, la contienda -que se convirtió en la más relevante a nivel nacional- era entre la expresidenta Cristina Fernández de Kirchner (Unidad Ciudadana) y el Ministro de Educación de Macri, Esteban Bullrich. Sin embargo, producto del poco nivel de conocimiento de Bullrich, quien encabezó la campaña fue la Gobernadora María Eugenia Vidal, dirigente de Cambiemos con mejor imagen nacional. De este modo, las principales figuras nacionales ${ }^{4}$ que se incluyen en este estudio son: Cristina Fernández de Kirchner (Unidad Ciudadana), Elisa Carrió (Cambiemos), Miriam Bregman (FIT) y María Eugenia Vidal (Cambiemos).

En la provincia de Mendoza el calendario electoral fue el mismo que a nivel nacional, aunque se vislumbran diferencias respecto de la composición de las listas. La alianza provincial que representa a Cambiemos se denominó Cambia Mendoza y llevó a una mujer como cabeza de lista, la exministra de salud de la provincia Claudia Najul. En tanto, el FIT también presentó a una política al frente, la exlegisladora provincial Noelia Barbeito. La representación local del Partido Justicialista la encabezó un hombre, Omar Félix, aunque la segunda fue Cecilia Juri, una dirigente que compitió en las PASO por el kirchnerismo ${ }^{5}$ local. Finalmente, se incluye en el análisis a la dirigente justicialista -no kirchneristaPatricia Fadel, quien fue una de las principales figuras aliadas a Omar Félix y es una dirigente histórica del justicialismo provincial. En síntesis, las políticas analizadas de Mendoza son: Claudia Najul (Cambia Mendoza), Cecilia Juri (Somos Mendoza), Noelia Barbeito (FIT) y Patricia Fadel (Partido Justicialista).

La decisión de incluir figuras políticas que fueron candidatas junto a otras que no lo fueron radica en que la disparidad de género es un problema que la política nacional arrastra desde hace años. Si bien hubo grandes avances mediante leyes de cupo femenino ${ }^{6}$, aún es difícil hallar mujeres cabeza de lista. Dado que el propósito central del trabajo es analizar el desempeño de dirigentes mujeres en Twitter, se eligieron las figuras mujeres más relevantes con incidencia real en las campañas de la Ciudad de Buenos Aires, de la Provincia de Buenos Aires y de Mendoza.

Las elecciones definitivas se llevaron a cabo el 23 de octubre de 2017. Ese día, Esteban Bullrich se impuso $(41,35 \%)$ a Cristina Fernández $(37,31 \%)$ en la provincia de Buenos Aires. Elisa Carrió venció en la Ciudad de Buenos Aires (50,97\%) a Daniel Filmus (21,74\%). En tanto, Miriam Bregman logró un escaño en la

4 Se las considera figuras nacionales ya que al ser candidatas y/o funcionarias de la Ciudad de Buenos Aires y de la Provincia de Buenos Aires tienen altos niveles de conocimiento y proyección nacional. 5 Kirchnerismo se utiliza en la jerga política argentina para referir al movimiento que adhiere al ciclo político inaugurado por el expresidente Néstor Kirchner (2003 - 2007) y la expresidenta Cristina Fernández (2007 - 2015).

6 Ver Ley de Cupo Femenino No 24.012/91: http://servicios.infoleg.gob.ar/infoleglnternet/anexos/04999/411/norma.htm 
legislatura porteña (6,87\%). En la provincia de Mendoza Claudia Najul se alzó con la victoria $(45,73 \%)$ contra la dupla Felix - Juri $(25,38 \%)$. En tanto, Noelia Barbeito no consiguió una banca en el Congreso Nacional $(11,69 \%)$.

\section{Marco Teórico}

La red social Twitter data de 2006. Su particular formato basado en textos cortos (140 caracteres que en 2017 se duplicaron a 280) asimilables a los mensajes de texto de los teléfonos móviles generó una serie especulaciones acerca de cómo definirla. Enrolada dentro de la familia de Redes Sociales (RRSS), Twitter se define como una red de microblogging que, a partir de la acción de tuitear, permite propagar un mensaje espontáneo y breve con afán de generar un flujo de opinión instantánea en vivo (Van Dijk, 2016). En ese sentido, también es considerado como un nuevo medio de difusión de información (Kwak et al., 2010).

El objetivo declarado de su creador, Jack Dorsey, era convertir a la plataforma en un servicio público indispensable pensado para posibilitar conversaciones libres y aleatorias que sean caja de resonancia de la opinión pública. Sin embargo

las cañerías de Twitter no transportan solo torrentes de tuits en vivo, ni la plataforma ni sus usuarios son meros portadores de información. Por el contrario, en ellas se generan flujos de datos con el objetivo de promover ciertos usos y usuarios por encima de otros (...) debido a la presión existente por volver redituables sus contenidos (Van Dijk, 2016: 116).

En términos de Parisier (2017), las redes sociales -incluida Twitter-, producto de los algoritmos que las regulan, constituyen una suerte de burbujas de filtro, agrupando a los moradores virtuales en comunidades amalgamadas por gustos, intereses y posturas comunes que reflejan, a la vez que (re)definen, sus identidades. En el mismo sentido, Calvo (2015) sostiene que los algoritmos seleccionan del total un número reducido de tuits que aparecen a la carta en cada timeline, ajustados a las preferencias previas de los tuiteros. Así, los tuits elegidos dan lugar a "una cámara de eco, un espacio virtual donde la información que recibimos es un eco de nuestros propios prejuicios y de nuestras creencias sobre cómo funciona el mundo" (Calvo, 2015: 15).

El resultado es que las características estructurales de la plataforma -el algoritmo y la búsqueda de monetización de los contenidos- contribuyen con la construcción de una serie de barrios virtuales cuyas agendas suelen ser consistentes con los puntos de vista de sus miembros, sobre todo en lo concerniente a asuntos políticos (Barberá et al., 2015).

El complemento del funcionamiento algorítmico está constituido por procesos de atención selectivas de los usuarios, quienes se acercan a información congruente con sus creencias y valores (Bizer et al., 2006). A través de sus búsquedas, posteos, likes y retuis trazan un recorrido en la red que da cuenta de activos 
procesos de selección de contenidos que son luego retroalimentados por la plataforma.

Otras de las características de la tuitósfera, contrariamente a algunas creencias, es que no es un espacio democrático. Está dominada por una serie de cuentas que funcionan como "formadoras de opinión" (Calvo, 2015: 45) o "autoridades" (Calvo y Aruguete, 2018: 203) que marcan el pulso de la comunidad en la cual se insertan. Estas figuras, generalmente cuentas institucionalizadas de políticos destacados, periodistas, líderes de opinión e incluso medios tradicionales, logran un volumen de diseminación mucho mayor que el de cualquier ciudadano común. Si bien hay excepciones comprobadas de "influencers" que lograron a través de Twitter construir cuentas con volúmenes importantes de seguidores, no son los casos más frecuentes. La red se completa con un volumen mayor de "seguidores" (hubs) que tienden a interactuar con las autoridades de sus comunidades y a viralizar los contenidos que estas producen.

En ese complejo ecosistema pululan una serie de cuentas conocidas como trolls, es decir, individuos que "de forma anónima se dedican a distorsionar la comunicación de usuarios de una misma comunidad" (Calvo, 2015: 105); y bots, otras cuentas que con los mismos objetivos interactúan de manera automatizada. Ambas figuras tienden a ser promovidas por espacios políticos generalmente institucionalizados que, a través de estas herramientas digitales, intentan moldear la opinión pública dentro de las comunidades en red. Dato no menor si se tiene en cuenta que a partir de los hashtags Twitter marca minuto a minuto una serie de tendencias (Trending Topics) que son parte de las ambiciones de la plataforma de "medir, diseñar y movilizar al mismo tiempo los estados de ánimo del público" (Van Dijk, 2016: 131).

Ahora bien, si Twitter es considerada como una red social que en ocasiones funciona como altavoz de la opinión pública y fuente de información a la vez porque para algunos usuarios es más valiosa la información que obtienen de la tuitósfera que de los medios tradicionales como la radio, televisión y prensa escrita (Bode y Dalrymple, 2016)- cabe preguntarse: ¿pueden las redes sociales disputar la agenda mediática a los medios tradicionales?

La respuesta a esta pregunta adquiere gran relevancia, puesto que lo que está en juego, en última instancia, son las premisas básicas de la Teoría de la AgendaSetting. Esta perspectiva, acuñada por McCombs y Shaw (1972), estipula que los medios de comunicación obtienen relativo éxito en decirle a la gente sobre qué temas pensar y cómo pensar sobre ellos (McCombs et al., 1997). Si los públicos, a través de redes sociales, blogs, o medios digitales, pudieran dialogar entre sí, compartiendo temarios y atributos por fuera del circuito periodístico, las hipótesis de la Agenda-Setting perderían parte de su potencia. 
Los entornos virtuales promovieron el interrogante sobre si frente a los modos actuales de producción, circulación y consumo de contenidos segmentados y "a la carta", los medios seguirían manteniendo la capacidad de instalación de temas en la opinión pública.

Así, surgió una nueva línea de investigación, la Agenda-Melding (fusión de agendas) (McCombs y Valenzuela, 2014).Partiendo de los conceptos de exposición selectiva (Klapper, 1960) y de disonancia cognitiva (Festinger, 1957), Shaw et al. (1999) afirman que los sujetos se ven motivados a agruparse en comunidades según sus puntos de vista, creencias y prácticas compartidas. Este comportamiento, que las redes sociales refuerzan mediante algoritmos, demuestra que en la comunidad ya no domina una única agenda temática, sino que, más bien, corresponde hablar de comunidades que conforman un sistema social que "se puede describir como una asamblea de agendas competitivas" (Shaw et al., 1999: 20).

En resumidas palabras, los sujetos "pertenecen a colectivos sociales para evitar el dilema de vivir en el aislamiento intelectual. Así, las personas funden sus agendas con las de sus pares y permiten que la congruencia social y cognitiva facilite el establecimiento de agenda" (Calvo y Aruguete, 2018: 195).

En la esfera pública virtual, esto significa que los tuiteros prefieren seguir a los políticos, medios y cuentas afines con sus formas de ver el mundo. Y de este modo, "dialogan y funden sus agendas con aquellos que pasean por sus mismos barrios" (Calvo y Aruguete, 2018: 195), forman parte de sus mismas burbujas (Parisier, 2017) o comunidades virtuales (Calvo, 2015).

Para analizar la relación entre agendas mediáticas y Twitter, algunos trabajos empíricos resultan reveladores. Una investigación desarrollada en España tuvo como objetivo analizar si los temas más discutidos en la red social Twitter tenían relación con la agenda propuesta por los medios más importantes del país. El estudio halló una fuerte correlación entre los asuntos destacados por los portales de noticias de los diarios El País y El Mundo y los comentarios de los Twitteros españoles (Rubio, 2014), por lo cual los medios tradicionales, aún en su versión digital, conservarían la capacidad de instalar ciertos temas que luego son discutidos en la esfera pública digital.

Otra investigación halló que, en la Argentina, respecto de la muerte del fiscal Alberto Nisman ${ }^{7}$ en 2015, de los diez tuits que más circularon, tres fueron originados por el diario La Nación. Además, en el podio de los más influyentes se destacaron las cuentas de los medios tradicionales, las que reprodujeron un estado de polarización presente en el entramado social. El estudio demuestra

7 Alberto Nisman fue un Fiscal Federal de la Nación que apareció muerto en su departamento. 
que, si bien en Twitter los usuarios interactúan de manera individual con las fuentes de información, la red es muy jerárquica y sólo algunos posteos suelen ser exitosos. Y de este selecto grupo, muchos corresponden a cuentas -y contenidosde medios consolidados.

En relación con los estudios de mujeres políticas, investigaciones previas han concluido que, si bien la red social Twitter les sirve a las candidatas para transmitir su fortaleza como mujeres a través de la activación y desactivación de estereotipos ligados al género femenino (López y Vallejo, 2016) y la refutación de otros relativos a la superioridad del hombre en los cargos directivos (McGregor, Lawrence y Cardona, 2017), sus twits se insertan en una estructura machista que es reproducida por las redes sociales (McGregor y Mourão, 2016), disminuyendo su visibilidad y capacidad de respuesta frente a la actividad de los dirigentes varones en la red. De este modo, se instituye una brecha de género virtual (Almansa, Smolak y López, 2018) que aún no ha podido ser superada.

\section{Objetivos e hipótesis}

En términos generales se pretende analizar el uso de la red social Twitter por parte de las principales lideresas políticas de Buenos Aires y Mendoza en el contexto de la campaña electoral 2017. Específicamente, se procura estimar la frecuencia de tuits de cada una de las políticas, indagar si la variable "género" influye sobre los temas más recurrentes en sus tuits, describir las interacciones entre ellas, entre ellas y los medios tradicionales y entre ellas y sus seguidores; y analizar la influencia de la variable geográfica en el contenido y circulación de los posteos.

Asociadas a estos objetivos y en relación con el marco teórico propuesto se estructuran las siguientes hipótesis:

H1: La frecuencia de tuits es mayor en aquellas políticas con mayor nivel de institucionalización.

H2: Entre los temas más recurrentes difundidos por las candidatas se encuentran algunos asuntos referidos a problemáticas de género.

H3: Las candidatas interactúan principalmente con otras políticas y medios afines.

H4: Producto de la estructura de Twitter, que tiende a construir comunidades, la variable geográfica se expresa principalmente en el nivel temático de los tuits.

\section{Metodología}

La estrategia metodológica se desarrolla en función de los objetivos expuestos. El dataset que se extrajo usando datos públicos de Twitter fue dependiente de las candidatas que formaron parte del presente análisis. A continuación se presentan los detalles sobre la recolección de los datasets empleados, así como la metodología aplicada para obtener los resultados. Se explican las decisiones tomadas sobre la extracción de datos y los pasos de preprocesamiento para limpiarlos o prepararlos. Esto facilita la reproductibilidad de los experimentos. 
Además, se proporcionan detalles del uso de teoría de grafos como herramienta para caracterizar a las mujeres en estudio.

Tabla 1. Número de tuits y retuits de las lideresas políticas para los cuatro meses en estudio

\begin{tabular}{|c|c|c|}
\hline Cuenta de Twitter & NumTuits & NumRetuits \\
\hline @CFKArgentina & 760 & 536 \\
\hline @elisacarrio & 486 & 158 \\
\hline @myriambregman & 867 & 2177 \\
\hline @ClauNaj & 270 & 377 \\
\hline @fadelpatricia & 125 & 101 \\
\hline @barbeitonoelia & 461 & 342 \\
\hline @mariuvidal & 57 & 4 \\
\hline @CeciJuri_K & 265 & 273 \\
\hline
\end{tabular}

Fuente: elaboración propia

\subsection{Datos}

Los conjuntos de datos, también llamados datasets, se extrajeron teniendo en cuenta la lista de mujeres argentinas consideradas referentes en la política en cada una de las localidades analizadas. Específicamente, se tomó en cuenta a las candidatas para las elecciones legislativas de octubre de 2017. Los datasets representan la actividad de las lideresas en Twitter para los meses de julio, agosto, septiembre y octubre de 2017. La Tabla 1 muestra los nombres de sus cuentas, así como el número de tuits y retuits analizados para ese período de tiempo. Vale la pena mencionar que se utiliza el término "usuarios" para referirnos a las mujeres en el estudio (término más general y técnico).

\subsection{Recopilación}

Los tuits de los usuarios se recopilaron utilizando la API de Twitter (Application Public Interface) que es la interface que ayuda a los desarrolladores de programas a conectarse a las bases de datos de Twitter para la recolección de los datos (siempre que estos sean públicos). El código fue desarrollado en Python, tanto para la etapa de recolección de tuits como para su procesamiento. 
Se recopilaron las publicaciones presentes en los perfiles del usuario correspondiente (timelines) a través de su nombre de usuario en Twitter (es decir, @usuario) $^{8}$.

De acuerdo a las normas de uso del API de Twitter, éste permite la extracción de los últimos 3200 tuits + retuits publicados por el usuario. Sin embargo, como se dijo anteriormente, se filtraron los tuits/retuits publicados durante el período del 1 de julio al 31 de octubre de 2017. Cabe mencionar que la extracción de datos fue realizada semanalmente, desde la primera semana del mes de julio hasta la última semana del mes de octubre, teniendo en cuenta el proceso electoral. A manera de control, se verificó que no exista pérdida ni duplicidad de datos a través de la validación del identificador o 'id' de los tuits. De igual forma, se consideró la fecha de creación de los tuits y retuits para verificar cuándo fue creado el post.

\subsection{Preprocesamiento}

Como parte del análisis, se tomaron en cuenta los hashtags publicados por los usuarios. La motivación para esto es que el uso de etiquetas o hashtags otorga algún significado relevante en el nivel temático (Zappavigna, 2015) y estructura las publicaciones de los usuarios. Dicho tipo de categorización se conoce como folksonomy (forma social de clasificación de contenido). Sin embargo, es común encontrar variaciones o malas prácticas en el uso de un mismo hashtag. Por ejemplo, algunos usuarios pueden escribirlo con letras mayúsculas, usando acentos, o podemos encontrar espacios en blanco faltantes entre ellos (\#VAMOSFernández, \#noLeymordaza \#Unidosmendoza).

Dadas estas condiciones, se necesita un procesamiento previo de hashtags antes de realizar el análisis de las publicaciones del usuario. Para hacerlo, una vez extraída la lista de tuits del usuario (y todos los metadatos relacionados con cada tuit), se recopila la lista de hashtags presentes en el tuit. Luego se modifican los hashtags de los usuarios de tal manera que exista una representación estándar.

En general, los hashtags se establecieron en minúsculas y se excluyó el uso de caracteres especiales (se eliminaron los acentos latinos en las palabras). Como resultado, se normalizó el uso de hashtags para poder identificar automáticamente a los usuarios que hablan sobre los mismos asuntos.

La distribución de probabilidad de los hashtags presenta una estructura long tail, que muestra que hay un gran número con una frecuencia de uso de 1 (el hashtag aparece en uno o dos tuits en los cuatro meses de análisis, (Kwak et al., 2010)).

Debido a la distribución se ha decidido aplicar la ley Power Law para el análisis de los datos, es decir que se obtiene $80 \%$ de hashtags con frecuencia de 1 o 2 y $20 \%$

8 https://developer.Twitter.com/en/docs/tweets/timelines/api-reference/get-statuses-user_timeline 
de hashtags que presentan mayor frecuencia de uso. En otras palabras, para el estudio de la actividad política de los usuarios se han considerado únicamente los que se encuentran en la "cabeza" de la distribución (20\%) y se han descartado los que se ubican en la "cola" o long tail.

Tabla 2. Ejemplo de Grafo Dirigido y con Peso

\begin{tabular}{ccc}
\hline Nodo de Origen & Nodo de Destino & Frecuencia de Uso \\
$@$ CFKArgentina & ahoraunidadciudadana & 76 \\
$@$ CFKArgentina & cristinaenracing & 32 \\
@CeciJuri_K & unidadciudadana & 85 \\
@CeciJuri_K & somosmendoza & 61 \\
@barbeitonoelia & niunamenos & 14 \\
@barbeitonoelia & debateenel9 & 12 \\
\hline
\end{tabular}

Fuente: elaboración propia

\subsection{Perfil de los usuarios mediante el empleo de Grafos}

Para poder representar de forma visual la relación entre las candidatas y el uso de hashtags se creó un grafo dirigido con pesos (weighted directed graph), con el usuario o candidata como nodo de origen, el hashtag (utilizado al menos 3 veces por el usuario) como el nodo de destino y su frecuencia de uso como el peso (Bellaachia y Al-Dhelaan, 2012).

La Tabla 2 muestra un ejemplo de cómo se estructura el grafo. La herramienta que ayuda a administrar grafos en Python es NetworkX. Al utilizarla, se creó el grafo descrito, el cual será de ayuda para definir las interacciones entre los usuarios en base al uso de hashtags. En el caso de la representación visual del grafo, se utilizó la herramienta Gephi. Esta es capaz de leer los grafos generados por NetworkX y representarlos visualmente; es decir, permite el uso de colores y tamaños para representar los nodos. Además, para detectar comunidades donde los nodos forman grupos por afinidad de uso de hashtags y las correspondientes conexiones, Gephi tiene la opción de usar el algoritmo Louvain de detección de comunidades (Blondel et al., 2008). El algoritmo de detección de comunidades agrupa los nodos que están fuertemente conectados; y dado el tipo de grafo empleado, también usa los pesos proporcionados para llegar a definir las comunidades.

\section{Resultados}

De acuerdo con los objetivos específicos de este trabajo, en primer lugar, se revelan algunas características básicas de las cuentas verificadas de las lideresas políticas. 
Fuente: elaboración propia

Seguidores en Twitter

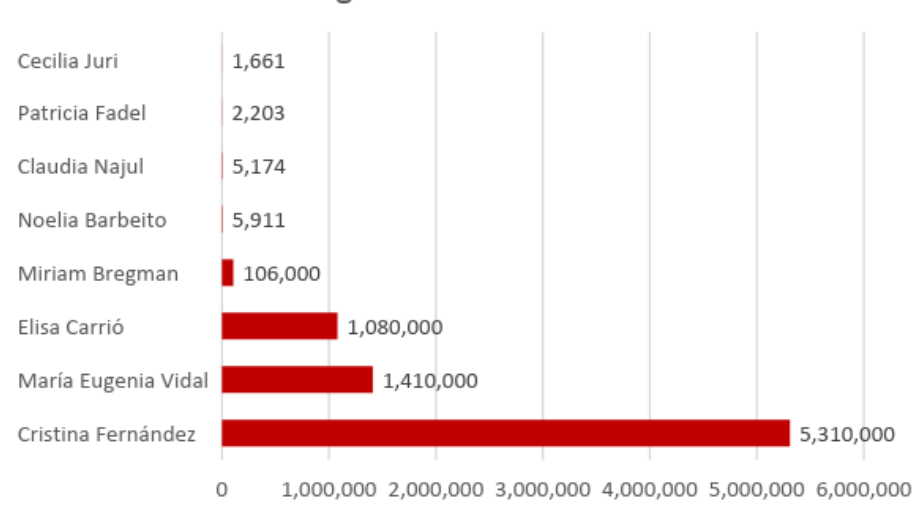

Gráfico 1. Seguidores en Twitter lideresas políticas

El primer dato que surge del análisis es la abrumadora diferencia de seguidores entre las políticas de Buenos Aires y las de la provincia de Mendoza. Si bien Cristina Fernández quintuplica en followers a Elisa Carrió, en buena medida esto se debe a la popularidad que le dio haber pasado por la presidencia de la nación. No obstante, aún con sustanciales diferencias entre ellas, las políticas de Buenos Aires logran una presencia en la red que es mucho mayor que la de las políticas del interior, generalmente sólo reconocidas en sus comarcas. En este sentido, es posible afirmar la incidencia de la variable geográfica en el nivel de seguidores que obtienen las mujeres políticas en Twitter.

Fuente: elaboración propia

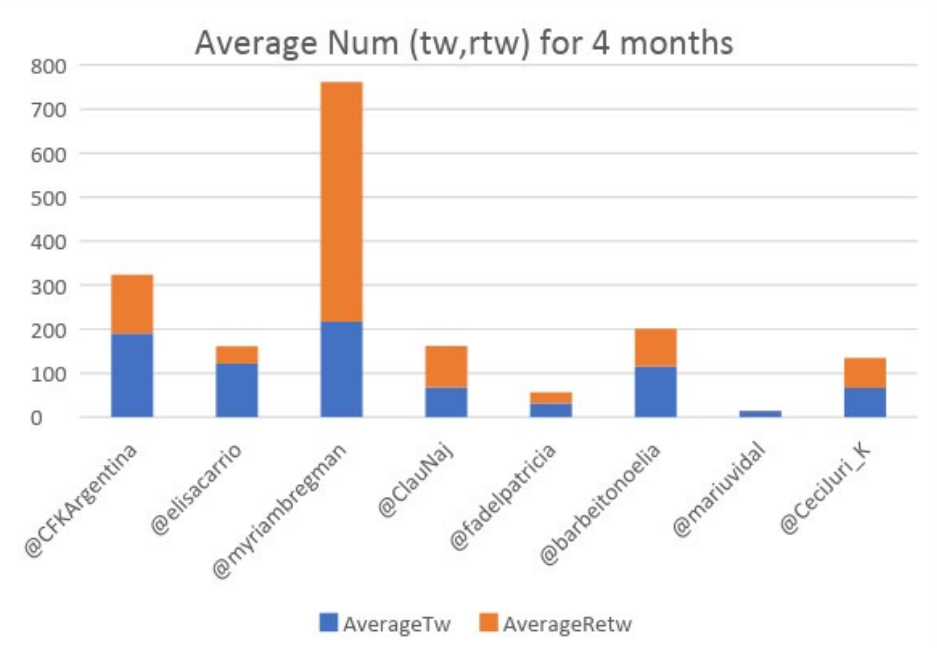

Gráfico 2. Promedio de tuits y retuits Julio - octubre de 2017. 
Otra de las intenciones de este trabajo era analizar la frecuencia de utilización de la red social Twitter por parte de las políticas argentinas. El gráfico 2 permite advertir que el uso no está necesariamente ligado a la cantidad de seguidores. Los datos demuestran que la cuenta más activa fue la de Miriam Bregman, quien está lejos de ser la que mayor cantidad de contactos tiene. En cambio, tanto Cristina Fernández como Elisa Carrió evidenciaron, pese a tener más followers, un uso menor de la red social. Esto coincide con el planteamiento que realiza Jungherr (2016) cuando afirma que los candidatos de oposición parecen más propensos a utilizar Twitter que los del gobierno.

Otro hallazgo importante es que la variable geográfica pareciera no impactar en la frecuencia de tuits. Los promedios de actividad, salvo las excepciones mencionadas, fueron parejos entre las lideresas, independientemente del lugar de residencia y/o de desempeño político.

Si se pone el foco el análisis de las particularidades de la cantidad de tuits y retuits según cada dirigente, es posible advertir que una serie datos expresan que el uso de la red no es uniforme. Mientras que la composición de los tuiteos de Cristina Kirchner y Carrió son de generación propia en su mayoría9 (190 y 121,5 por mes respectivamente), la diferencia que marca Miriam Bregman, cuya actividad duplica y triplica al resto, es que sus retuis $(544,25)$ son muchos más que sus tuits $(216,75)$. Es decir, la proporción de material tercerizado con el que la candidata acuerda -y difunde- es mayor que los contenidos que ella misma genera.

Otro dato importante es el alto nivel de participación de Noelia Barbeito. Perteneciente al mismo espacio que Bregman (FIT), alianza de partidos trostkistas menor en cantidad de votos, ambas candidatas tuvieron mucha participación en Twitter como estrategia de campaña, incluso muy por encima de lideresas más conocidas o provenientes de partidos tradicionales.

Finalmente, llama la atención la casi nula actividad de la cuenta de María Eugenia Vidal (Cambiemos). Su espacio político se mostró pionero en el uso de Big Data y redes sociales en campañas electorales. La poca actividad de Vidal contradice incluso su alta visibilidad mediática y territorial durante toda la contienda electoral, apuntalando una fórmula desconocida para los electores. En ese sentido, la menor cantidad de tuits respecto incluso de su compañera de espacio, Elisa Carrió, evidencia un uso diferente de las redes dentro del mismo partido político.

9 Al referirnos a "generación propia" queremos decir que son contenidos originales de la cuenta y no material producido por otras cuentas, medios o sitios, independientemente de si la cuenta es administrada por community managers o por las propias lideresas, aspecto que escapa a los alcances de este estudio. 
Ahora bien, otro de los objetivos particulares de este trabajo se proponía indagar sobre los tópicos promovidos por las lideresas. Para ello se analizaron los hashtags más frecuentes y su perduración en el tiempo.

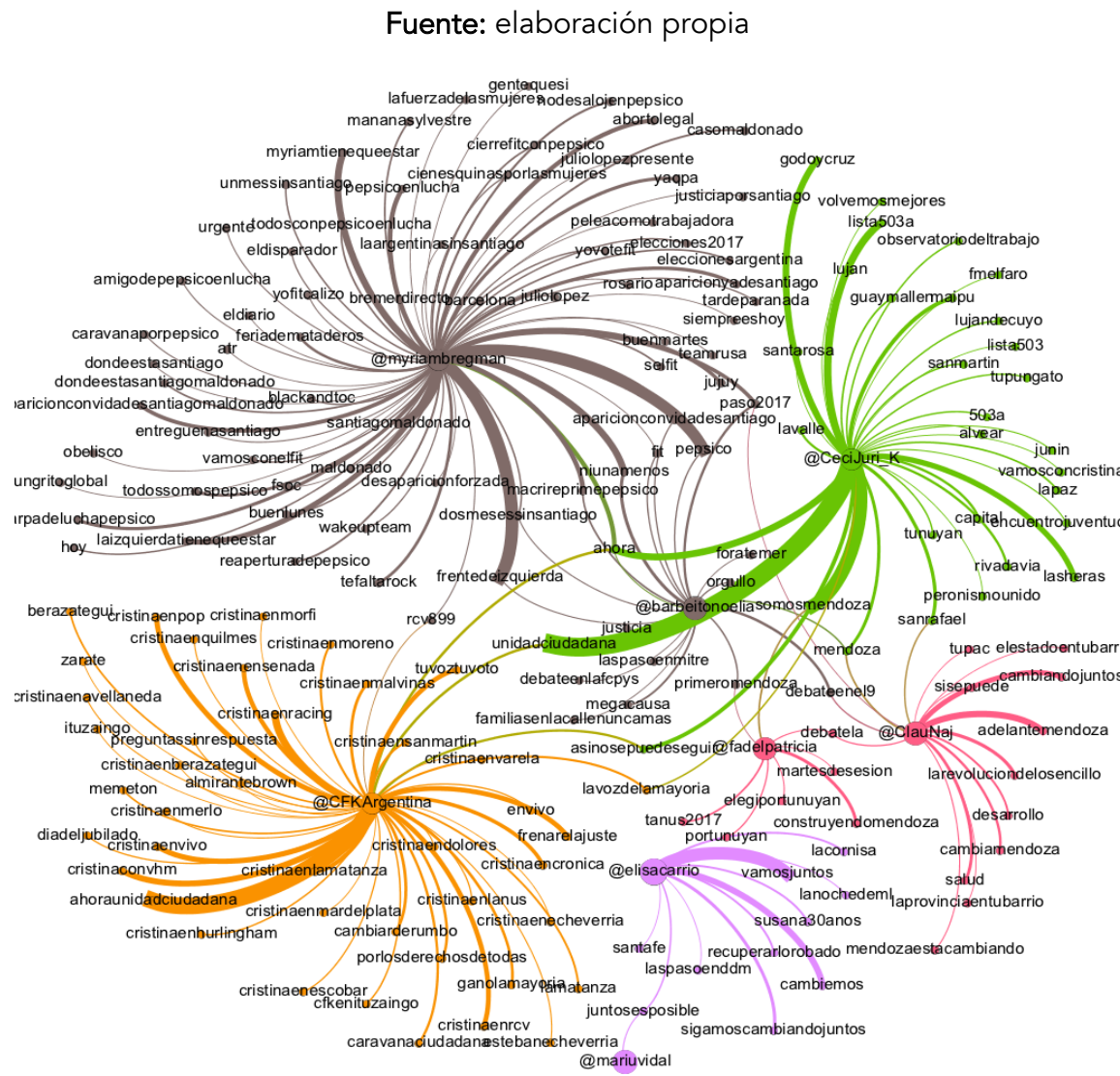

Gráfico 3. Hashtags y comunidades en Twitter. Lideresas políticas argentinas.

El gráfico 3, que representa los hashtags, su densidad de uso y las interacciones que se dieron a partir de ellos, permite desarrollar en simultáneo diferentes niveles de análisis ligados con los objetivos planteados.

En primer lugar, se analiza cuáles fueron los temas más desarrollados por cada una de las políticas. Un primer hallazgo es que todas utilizaron asiduamente hashtags ligados a sus consignas de campaña. \#AhoraUnidadCiudadana en el caso de Cristina Fernández, \#FrenteDelzquierda y \#MiriamTieneQueEstar en el caso de Miriam Bregman, \#Cambiemos y \#VamosJuntos en el caso de Elisa Carrió, \#UnidadCiudadana y \#SomosMendoza en el caso de Cecilia Juri, \#CambiaMendoza y \#AdelanteMendoza en el caso de Claudia Najul y \#ConstruyendoMendoza en el caso de Patricia Fadel, fueron las etiquetas más utilizadas. 
Este hallazgo es coincidente con la propuesta de Jungherr (2016) que sostiene que en periodo de elecciones habitualmente los mensajes de los candidatos están íntimamente vinculados a su estrategia de comunicación política y que utilizan la plataforma como un medio para la difusión de su agenda de actividades de campaña, más que para conversar con sus seguidores.

Una de las particularidades de las políticas mendocinas es que la etiqueta "Mendoza" estuvo presente en cada uno de los hashtags más tuiteados. Así, resulta posible afirmar que, en la conformación de las comunidades virtuales, la fijación de las consignas y la mención explícita de la localidad funcionan en Twitter como filtros que permiten no sólo dirigir el mensaje, sino fijarlo territorialmente.

Es dable destacar que los principales tuits de Noelia Barbeito, referente del FIT mendocino, estuvieron más asociados a temas, como \#NiUnaMenos y \#Pepsico. El primer hashtag hace alusión a una campaña nacional e internacional contra la violencia machista. El segundo, a una dura represión que tuvo lugar en la fábrica alimenticia Pepsico durante la campaña, en la que los principales dirigentes gremiales eran del FIT y de la izquierda. Entonces, la dirigente del FIT marcó una diferencia al no fijar sus tuits al territorio, subiéndose a una agenda temática nacional.

De todos modos, las burbujas de filtro (Parisier, 2017), en términos generales, no recortan sólo por sector político, también lo hacen por región. Es decir, más allá de la excepción descripta, se comprueba la hipótesis referida a la influencia de la dimensión territorial sobre la actividad en Twitter, ya que la necesidad de fijación de la dimensión territorial en el uso de las etiquetas recorta un público objetivo y un radio de acción que coincide con votantes concretos.

Otra dimensión de análisis consistía en detectar cuáles fueron los temas salientes de cada sector político y si existieron puntos de contacto entre las lideresas. La evidencia empírica demuestra que el uso de Twitter por parte de las candidatas consistió mucho más en difundir consignas y acciones territoriales o mediáticas que en tratar grandes asuntos de preocupación nacional, salvo algunas excepciones.

Los hashtags más salientes de Cristina Fernández fueron del tipo \#CristinaEnRacing (estadio en el que se llevó a cabo su cierre de campaña el 16 de octubre de 2017) o \#CFKEnltuzaingó (localidad del oeste bonaerense en el que se desarrolló un acto de campaña). En relación con lo estrictamente temático, el único asunto que aparece con frecuencia en sus tuits es \#FrenarElAjuste, referido a un discurso que enfrenta las políticas económicas de recorte de gastos del Estado del presidente Mauricio Macri. Otro dato saliente para esta candidata es que fue la que más relación con los medios tradicionales evidenció en su uso de Twitter. Así como muchos de sus posteos anunciaban su presencia en 
diferentes localidades, otros hashtags como \#CristinaConVHM, \#CristinaEnCrónica o \#CristinaEnPop anunciaban la asistencia a diferentes programas de radio y TV a los que les otorgó entrevistas exclusivas, dato no menor luego de años en que como presidenta casi no tuvo contacto directo con los medios de comunicación. Finalmente, resulta interesante analizar que la problemática propia de género estuvo ausente en el discurso tuitero de Cristina Kirchner.

El caso de Elisa Carrió resulta similar. Si bien la dirigente no incluyó en los tuits sus recorridas de campaña, el uso de la red se limitó a una serie de consignas partidarias como \#VamosJuntos y \#Cambiemos. En relación con los medios tradicionales de comunicación, \#LaCornisa linkea la tuitósfera con un ciclo periodístico televisivo afín a las ideas de Cambiemos. A nivel temático, el tópico más fuerte que aparece es \#RecuperarLoRobado y hace referencia a la supuesta corrupción de la década kirchnerista. Finalmente, la cuestión de género también estuvo ausente en su discurso virtual.

Si se analiza el uso de Twitter de Miriam Bregman aparecen las primeras diferencias. Si bien, como el resto de las candidatas, utilizó consignas partidarias, el caso particular se muestra diferente en varios aspectos. En primer lugar, porque las referencias múltiples a la represión en \#Pepsico puso en su agenda el tema del trabajo y la protesta social. El segundo aspecto para destacar es la presencia de tópicos relacionados con el tema género. \#NiUnaMenos y \#AbortoLegal dan cuenta de dos reivindicaciones centrales del movimiento feminista que solo manifestó en campaña la izquierda. Del mismo modo, se detectaron diferentes hashtags sobre \#SantiagoMaldonado, un joven artesano militante desaparecido durante una represión policial en la provincia de Chubut el 1 de agosto de 2017, donde estaba defendiendo posesiones territoriales de pueblos originarios Mapuches.

El caso, que culminó con la aparición del cuerpo flotando en un río el 17 de octubre, se incorporó a la discursividad del FIT a partir de la puesta en agenda de la problemática de los Derechos Humanos. También se detectan en Bregman referencias a actos de campaña a partir de sus ubicaciones en el territorio, como \#Obelisco, a la vez que existen relaciones de los tuits con medios tradicionales, principalmente para promocionar la presencia de la candidata en \#EIDiario, \#MañanaSilvestre y \#SiempreEsHoy, todos programas de radio y TV.

Ahora bien, a nivel provincial se comprueba que las dirigentes políticas dialogaron principalmente con las lideresas nacionales de los mismos espacios políticos, evidenciando la centralidad de Buenos Aires en la vida política nacional. Para ello el caso de Noelia Barbeito resulta elocuente. El grafo demuestra conexiones con su dirigente nacional, Miriam Bregman, a nivel temático a partir de \#Pepsico, \#SantiagoMaldonado y \#NiUnaMenos y a través de referencias partidarias con \#FrenteDelzquierda. La relación con los medios, en cambio, tiene raigambre local 
y se da con el hashtag \#DebateEnEl9, en referencia a un debate de candidatos que organizó el principal canal de TV de la provincia. Con esa etiqueta se detectan cruces incluso con candidatas de otros espacios políticos.

En el caso Claudia Najul, la candidata provincial de Cambiemos, las principales referencias fueron a consignas de campaña. A nivel temático sólo aparece \#Salud, seguramente porque es médica y se desempeñaba al frente del Ministerio de Salud de la provincia al momento de su candidatura. Finalmente, no hubo referencias al género en su discurso.

Lo mismo sucedió con la dirigente Justicialista Patricia Fadel, quien difundió consignas como \#ConstruyendoMendoza, hizo referencia a medios tradicionales con \#DebateLA y no dio lugar a temáticas de género en sus tuits.

Finalmente, Cecilia Juri, lideresa mendocina de la fuerza de Cristina Fernández, interactuó con ella a través de \#UnidadCiudadana y \#AsíNoSePuedeSeguir, hizo mucha referencia al territorio con hashtags como \#LasHeras, \#GodoyCruz o \#Tunuyán, todos departamentos de la provincia que recorrió en campaña, y se ocupó del tópico "trabajo" a partir de \#ObservatorioDelTrabajo, iniciativa local que dirigen profesionales ligados a su espacio político que monitorea la situación laboral en la provincia. Como en la mayoría de las candidatas, no existieron referencias a temáticas de género en su discurso virtual.

En suma, el análisis de los tuis de las lideresas políticas argentinas deja tres evidencias centrales que se ponen en diálogo con trabajos previos y que deberían contrastarse en futuros estudios. En primer lugar, se comprueba que las comunidades de cada una de ellas son principalmente cerradas, sin demasiados puntos de contacto por fuera de sus espacios políticos, aunque con asidua relación con medios tradicionales importantes para sus seguidores. En segundo lugar, se verifica que el factor geográfico es central para localizar la actividad, aún en espacios virtuales, aunque exista una importante referencia y centralidad de las políticas de Buenos Aires en las agendas locales. Finalmente, las mujeres políticas argentinas no han aprovechado en la campaña electoral 2017 la plataforma virtual para afirmar su condición de mujer o para establecer discusiones de género. Quienes lo hicieron, en mayor medida, fueron las representantes de la izquierda, en coincidencia con consignas históricas que sus partidos levantan desde aún antes de la existencia de la esfera pública virtual.

\section{Conclusiones}

A lo largo de este trabajo se analizó la presencia diferencial en Twitter de ocho lideresas políticas nacionales y provinciales con dispar nivel de conocimiento por parte del electorado y de penetración en las redes sociales. 
En relación con los objetivos planteados, uno de los primeros hallazgos estableció que la candidata del FIT de la Ciudad de Buenos Aires, Miriam Bregman, fue la que más utilizó la red social. Sin embargo, el porcentaje de retuiteos triplicó a los mensajes de producción propia. Las otras dos candidatas que más usaron la red fueron Cristina Fernández y Elisa Carrió. Los datos demostraron que no existe una relación necesaria entre uso y cantidad de seguidores, puesto que mayores niveles de actividad, como en el caso de las candidatas de izquierda, no correlacionan con millones de followers como poseen Fernández o Carrió, por ejemplo.

Asimismo, se puede observar que a pesar de que Internet se muestra como un espacio público que permite al candidato una relación más cercana con sus posibles votantes, Twitter es una plataforma que, algoritmos mediante, refuerza los vínculos ya existentes entre el líder político y sus seguidores y no genera nuevas relaciones con los indecisos o los electores de la oposición. Por lo tanto, debería ponerse en duda la afirmación de la potencialidad de la red social de modificar actitudes de voto (Bimber, 2003).

A la hora de medir la interacción entre las candidatas, el resultado del trabajo empírico dio cuenta que las burbujas de filtro (Parisier, 2017) impidieron cruces significativos en las narrativas de lideresas de diferentes espacios políticos. Más aún, casi no hubo diálogo entre ellas, ni de ellas con sus seguidores. Esto confirma que no se emplea la plataforma para generar interacción entre las líderes y los ciudadanos, sino que esta se convierte en un espacio informativo de su programa político y actividades de campaña (Bimber, 2003; Jungherr, 2016).

Las interacciones fueron, sobre todo, a partir de los asuntos y consignas que ellas promovieron a sus seguidores, lo que incrementa la endogamia de las burbujas. Asimismo, se evidencia un mayor nivel de diálogo entre políticas de un mismo espacio a nivel nacional y provincial, aunque sobre todo en este último nivel. La explicitación del territorio "Mendoza" genera un sub- recorte de las comunidades que ancla en lo territorial. Esta particularidad de generar burbujas temáticas, pero también locales, constituye otro hallazgo empírico que dialoga con otros estudios que les atribuyen a las comunidades de Twitter un carácter local (Aruguete, 2018; Calvo, 2015; Calvo y Aruguete, 2018; Parisier, 2017).

Un dato que no debe pasar desapercibido es el bajo nivel de desarrollo de narrativas políticas temáticas. Es decir, los tópicos centrales de la discusión política no pasaron por Twitter. Como ya se dijo, esta herramienta se utilizó casi únicamente para difundir actos de campaña tradicionales, como la presencia de las políticas en medios audiovisuales. De este modo, la potencialidad de Twitter de generar mensajes específicos que incluyan material escrito o audiovisual, de promover discusiones públicas en un formato propio y particular y de incluir narrativas transmedia a partir de la interacción con el público (Scolari, 2014) se 
descubre, como ya lo habían hecho D’Adamo, García y Kievsky (2015), subutilizada.

La interacción con medios tradicionales se halló en la mayoría de las candidatas, ya sea para publicitar apariciones, ya sea para difundir contenidos afines. En este sentido, la importancia que las lideresas le otorgaron a los medios tradicionales para difundir sus plataformas fue central, situación que pone en cuestión los vaticinios sobre su impotencia asociada al uso de las redes y el big data en las campañas políticas y fuera de ellas.

Por último, llama la atención la falta de relación entre políticas mujeres y problemáticas de género. Las únicas candidatas que pusieron el tema en agenda fueron Miriam Bregman y Noelia Barbeito. Probablemente debido a que este tipo de issues fueron históricamente patrimonio de los movimientos de izquierda. Es decir, la referencia a asuntos ligados a las mujeres en la campaña electoral en Twitter dependió mucho más de la propiedad de un sector político sobre determinado tema (Issue Ownership) (Ansolabehere y lyengar, 1994) que del género de las dirigentes que lo pusieron en agenda. 


\section{Referencias bibliográficas}

ALMANSA, A., SMOLAK, E. y LÓPEZ, P. (2018): La brecha política de género en las campañas internacionales en las redes sociales. En el $X$ Congreso Internacional Latina Camunicación Social (pp. 1-19). La Laguna (Tenerife): Sociedad Latina de Comunicación Social.

AMADO, A. y TARULLO, R. (2015): Tuitear para agendar: el uso de Twitter como gacetilla de prensa en la comunicación gubernamental. Revista Mexicana de Opinión Pública, v. 19, pp. 127-145.

ANSOLABEHERE, S. y IYENGAR, S. (1994): Riding the Wave and Claiming Ownership over Issues: The Joint Effects of Advertising and News Coverage in Campaigns. Public Opinion Quarterly, 58 (1), pp. 335-357, https://doi.org/10.1086/269431

ARUGUETE, N. (2018). \#2x1: diálogos al costado de la grieta. Intersecciones En Comunicación, v. 12, pp. 35-48.

BARBERÁ, P., JOST, J., NAGLER, J., TUCKER, J. y BONNEAU, R. (2015): Tweeting From Left to Right. Psychological Science, 26 (10), pp. 1531-1542.

BELLAACHIA, A. y AL-DHELAAN, M. (2012): Learning from Twitter hashtags: Leveraging proximate tags to enhance graph-based keyphrase extraction. IEEE International Conference on Green Computing and Communications, pp. 348357.

BIMBER, B. (2003): Information and American democracy: Technology in the evolution of political power. Cambridge: University Press.

BIZER, G., TORMALA, Z., RUCKER, D. y PETTY, R. (2006): Memory-based versus on-line processing: Implications for attitude strength. Journal of Experimental Social Psychology, 42 (5), pp. 646-653.

BLONDEL, V., GUILLAUME, J., LAMBIOTTE, R. y LEFEBVRE, E. (2008): Fast unfolding of communities in large networks. Journal of Statistical Mechanics: Theory and Experiment, 2 (10), doi:10.1088/1742-5468/2008/10/p10008.

BODE, L. y DALRYMPLE, K. (2016): Politics in 140 characters or less: Campaign communication, network interaction, and political participation on Twitter. Journal of Political Marketing, 15 (4), pp. 311-332.

CALVO, E. (2015): Anatomía política de Twitter en Argentina. Tuiteando \#Nisman. Buenos Aires: Capital Intelectual. 
CALVO, E. y ARUGUETE, N. (2018): \#Tarifazo. Medios tradicionales y fusión de agenda en redes sociales. Inmediaciones de La Comunicación, 13 (1), pp. 189213.

D’ADAMO, O., GARCÍA, V. y KIEVSKY, T. (2015): Comunicación política y redes sociales: análisis de las campañas para las elecciones legislativas de 2013 en la ciudad de Buenos Aires. Revista Mexicana de Opinión Pública, n. 19, pp. 107125.

FESTINGER, L. (1957): A Theory of Cognittive Dissonance. Stanford: Stanford University Press.

HABERMAS, J. (2006). Political communication in media society: Does democracy still enjoy an epistemic dimension? The impact of normative theory on empirical research. Communication Theory, 16 (4), pp. 411-426, https://doi.org/10.1111/j.1468-2885.2006.00280.x

HEIFETZ, R. (1997): Liderazgo sin respuestas fáciles: propuestas para un nuevo diálogo social en tiempos difíciles. Barcelona: Paidós Ibérica.

KLAPPER, J. (1960): The effects of mass communication. New York: Free Press.

JUNGHERR, A. (2016): Twitter use in election campaigns: A systematic literature review. Journal of Information Technology \& Politics, 13:1, pp. 72-91, doi: 10.1080/19331681.2015.1132401

KWAK, H., LEE, C., PARK, H. y MOON, S. (2010): What is Twitter, a Social Network or a News Media? Categories and Subject Descriptors. Ponencia presentada en International World Wide Web Conference Committee, pp. 1-10.

LÓPEZ, A. y VALLEJO, M. (2016): Femenine Stereotype in 140 Characters. Approach to the Hillary Clinton Campaign On Twitter. Revista de Comunicación, n. 15, pp. 48-69.

LUPANO, M. y CASTRO, A. (2008): Liderazgo y género. Identificación de Prototipos de liderazgo efectivo. Perspectivas en Psicología, 5 (1), pp. 69-77.

McCOMBS, M., LLAMAS, J., LÓPEZ, E. y REY, F. (1997): Candidate Images in Spanish Elections: Second-level Agenda-Setting Effect. Journalism and Mass Communication Quaterly, 74 (4), pp. 703-717.

McCOMBS, M. y SHAW, D. (1972): The Agenda-Setting Function of the Mass Media. Public Opinion Quarterly, v. 36, pp. 176-187. 
McCOMBS, M. y VALENZUELA, S. (2014): Agenda-Setting Theory: The Frontier Research Questions. Oxford.

McGREGOR, S., LAWRENCE, R. y CARDONA, A. (2017): Personalization, gender, and social media: gubernatorial candidates' social media strategies. Information Communication and Society, 20 (2), pp. 264-283.

McGREGOR, S. y MOURÃO, R. (2016): Talking Politics on Twitter: Gender, Elections, and Social Networks. Social Media and Society, 2 (3), pp. 1-14, https://doi.org/10.1177/2056305116664218

PARISIER, E. (2017): El filtro burbuja. Cómo la red decide lo que leemos y lo que pensamos. Barcelona: Penguin Random House.

RUBIO, R. (2014): Twitter y la teoría de la Agenda - Setting: mensajes de la opinión pública digital. Estudios Sobre El Mensaje Periodístico, v. 20, pp. 249264.

SCOLARI, C. (2014): Narrativas transmedia: nuevas formas de comunicar en la era digital. Anuario AC/E de Cultura Digital, pp. 71-81.

SHAW, D., McCOMBS, M., WEAVER, D. y HAMM, B. (1999): Individuals, Groups, and Agenda Melding: A Theory of Social Dissonance. International Journal of Public Opinion Research, 11 (1), pp. 2-24.

SINCA (2017): Encuesta Nacional de Consumos Culturales 2017. Buenos Aires.

VAN DIJK, J. (2016): La cultura de la conectividad. Una historia crítica de las redes sociales. Buenos Aires: Siglo XXI.

VARGO, C., GUO, L., McCOMBS, M. y SHAW, D. L. (2014): Network Issue Agendas on Twitter During the 2012 U.S. Presidential Election. Journal of Communication, 64 (2), pp. 296-316.

ZALLES, J. (2011): Liderazgo: Un concepto en evolución. Barcelona: Konrad Adenauer Stiftung.

ZAPPAVIGNA, M. (2015): Searchable talk: the linguistic functions of hashtags. Social Semiotics, 25 (3), pp. 274-291. 